\title{
Is thrombolysis for intermediate-risk pulmonary embolism beneficial? The case of Emeritus Professor Crow
}

\author{
Alessandro Squizzato $\cdot$ Lorenzo Moja \\ Gian Franco Gensini · Roberto Gusinu • \\ Andrea A. Conti
}

Published online: 9 June 2009

(C) SIMI 2009

\section{The methodologist's point of view}

\section{Alessandro Squizzato, Lorenzo Moja}

You are the attending physician on duty at the Emergency Department, when John Crow, a 69-year-old Emeritus Professor of cardiology comes with acute dyspnoea [1]. The day before he started complaining a right leg oedema and pain, he said. While starting to collect other clinical data and requiring tests you remember that time you met Dr. Crow on a luxurious conference. Was he the one supporting prescription of hormone replacement therapy to women on the mistaken basis that this would reduce the risk of heart? Dr. Crow's blood pressure is $120 / 80 \mathrm{mmHg}$. Spiral computed tomography shows emboli in more than

\section{A. Squizzato $(\square)$}

Department of Clinical Medicine, U.O. Medicina I, Ospedale di Circolo, University of Insubria, Viale Borri 57,

21100 Varese, Italy

e-mail: alexsquizzo@libero.it

\section{Moja}

Italian Cochrane Center, Mario Negri Institute

for Pharmacological Research, Milan, Italy

G. F. Gensini

Department of Critical Care Medicine and Surgery,

University of Florence and Azienda Ospedaliero-Universitaria

Careggi, Florence, Italy

R. Gusinu

DAI Cardiologico e dei Vasi Azienda Ospedaliero-Universitaria Careggi, Florence, Italy

\section{A. A. Conti}

Department of Critical Care Medicine and Surgery,

University of Florence and Don Carlo Gnocchi Foundation and IRCCS Florence, Florence, Italy
$50 \%$ of pulmonary vessels and a right ventricular enlargement. Blood troponin level is elevated and echocardiography confirm right ventricular enlargement.

The resident on duty, who is just back from an international meeting on venous thromboembolic diseases, is aware of the recently published European Society of Cardiology (ESC) guidelines and of the American College of Chest Physicians (ACCP) on this topic that suggest that absolute indications for thrombolysis are: massive pulmonary embolism with persistent hypotension or shock $[2,3]$. This is not your case. Your colleague reports that thrombolysis may be an option in selected cases of sub-massive pulmonary embolism (PE) defined as normal blood pressure and right ventricular dysfunction [2, 3]. This is your case. Indeed, before discussing with this declamated patient the convenient therapeutic strategy, you need to clarify the advantages and the risks of adding thrombolysis to heparin, which is your reference therapy. You have a look at the Cochrane Library and download the systematic review entitled Thrombolytic therapy for pulmonary embolism [4].

The Cochrane's point of view: a systematic review

Overall 679 patients were enrolled in 8 included randomised controlled trials [4]. Different types of thrombolysisalteplase, urokinase, streptokinase-were used and were compared with heparin alone. All trials excluded patients with high-risk PE, i.e. shock or persistent hypotension. Five of the eight trials had well reported and satisfactory methodological quality. Clinically sound outcomes were inconclusive: death rate odds ratio (OR) 0.89 [95\% confidence interval (CI) 0.4-1.78]; major haemorrhagic events OR 1.61 (95 CI 0.91-2.86). Thrombolytics improved some secondary outcomes-hemodynamic outcomes, perfusion 
lung scanning, pulmonary angiogram assessment and echocardiograms - to a greater extent than heparin alone. Against this background, the authors stated that they cannot conclude whether thrombolytic therapy added to heparin is better than heparin alone for PE based on the limited evidence found. They believe that more double-blind randomised controlled trials, focussing both on patients presenting with haemodynamically stable acute PE and on those patients with hemodynamic unstable condition, are required. The uncertainty facing you, if a patient with hemodynamic stable condition and sub-massive PE may benefit from thrombolysis, is still there.

What is the evidence in high-risk patients? Is evidence related to high-risk patients generalisable to intermediaterisk population? In the "Discussion" section, Cochrane authors underline that, until now, only one study has been published that compares thrombolytic therapy plus heparin versus heparin alone in hemodynamically unstable, high-risk PE patients [5]. Eight patients were randomised to receive either streptokinase plus heparin or heparin alone. The four patients who received streptokinase improved in the first hours after treatment and survived. All four patients treated with heparin alone died within few hours. This study is not included in the mortality meta-analysis, because the patients did not have confirmed diagnosis of PE and were only considered to have a high clinical suspicion of PE, therefore reviewers decided to exclude this study [4]. Although this decision may be in agreement with the review protocol and is legitimate, eventually authors did not consider four deaths in their qualitative and quantitative analyses (13\% of overall mortality incidence across trials), a valuable amount of information to drive clinical decision making.

\section{The PE panellist's point of view: clinical guidelines}

Two recently published guidelines both recognise that thrombolysis in PE patients without shock or persistent hypotension is still a debatable issue, but provide some recommendations [2, 3]. The ACCP panellists stated: "Thrombolytic therapy for PE remains controversial. The fundamental problem is that less than $800 \mathrm{PE}$ patients have been enrolled in randomised trials of thrombolysis plus anticoagulation versus anticoagulation alone" [2]. "In distinction to the last version of these guidelines that generally discouraged treatment of PE with thrombolytic therapy unless there was hemodynamic compromised, we suggest administration of thrombolytic therapy in selected high-risk patients without hypotension who are judged to have a low risk of bleeding. The decision to use thrombolytic therapy depends on the clinician's assessment of PE severity, prognosis, and risk of bleeding. For the majority of patients with $\mathrm{PE}$, we recommend against using thrombolytic therapy" [2].
The ESC, similarly, stated: "the overall effects of thrombolysis on the clinical outcome of patients with $\mathrm{PE}$ are difficult to assess" [3]. "In summary, routine use of thrombolysis in non-high risk patients is not recommended, but may be considered in selected patients with intermediate-risk PE and after thorough consideration of conditions increasing the risk of bleeding" [3]. The uncertainty facing you, if a patient with hemodynamic stable condition and sub-massive PE may benefit from thrombolysis, is still there.

The researcher's point of view: an ongoing trial

Because of the inadequacy of currently available data, in 2007 a European trial began enrolling patients with submassive PE [6]. Dr. Crow would be an ideal candidate for this trial: age 18 years or older; acute PE (first symptoms occurring 15 days or less before randomisation) confirmed by lung scan, or a positive spiral computed tomogram, or a positive pulmonary angiogram; right ventricular dysfunction confirmed by echocardiography or spiral computed tomography of the chest and a positive troponin I or T test. This trial will randomise approximately 1,000 patients to thrombolysis with a bolus regimen of tenecteplase plus heparin versus heparin alone. Planned sample size will possibly provide solid information on clinical outcomes, in particular death. Patients with PE in the enrolling centres are offered the opportunity to enter the trial: they can refuse to participate or accept that the 'fate' will decide their treatment. But in the $99 \%$ of hospitals all over the world, how should patients at intermediate risk treated? The uncertainty facing you does not wait.

The patients' point of view

You explore all caveats with Dr. Crow. Thrombolytic drugs are licensed for PE indication. Thrombolysis is the first-line therapy for high-risk PE, and heparin the first-line therapy for low-risk PE: risk of death is a continuum and, theoretically, some patients may benefit in the intermediate group. Evidence far too definitely suggests thrombolysis, but results on secondary outcomes are promising, although may be spurious. Therefore, you attempt to stratify Dr. Crow across high- and low-risk patients. Dr. Crow stays in between. You move to adverse events: major bleedings, in particular cerebral bleeding, are potentially thrombolysis-provoked harms.

Finally Dr. Crown feebly asks "If you were me, what treatment you will go with?" Walking toward the patient's room window, you discretely search a coin in your pocket while thinking that would have been a valuable effort by an emeritus professor to solve an important clinical question lasting since 15 years instead of proclaiming incautious 
opinions. Waiting for the future generation to produce facts it is not always an advantage.

\section{A clinician's point of view}

\section{Gian Franco Gensini, Roberto Gusinu, Andrea A. Conti}

Venous thromboembolism is a major clinical issue, determining up to $15 \%$ of in-hospital mortality, and accounting for up to $30 \%$ of deaths related to pregnancy and delivery in Europe and in the USA. Pulmonary embolism is responsible for mortality and complications related both to medical illnesses and surgical interventions, and its annual incidence ranges from approximately 20 to 70 cases per 100,000 persons [7]. Mortality rates largely depend on the severity of this clinical condition.

In the case reported above an apparently intermediaterisk patient affected by pulmonary embolism is described. Recent available clinical guidelines [2] clearly indicate that every pulmonary embolism patient should undergo quick risk stratification (grade $1 \mathrm{C}$ ) to address appropriate therapeutic management, and in people with hemodynamic compromise thrombolytic therapy is recommended in the absence of major contraindications (grade 1B). The authors of these guidelines also suggest the adoption of thrombolytic therapy in the majority of pulmonary embolism patients (grade 1B), clearly stating that a clinical evaluation which carefully considers the severity, the prognosis and the risk of haemorrhage in persons with pulmonary embolism has a key role in shaping the operative decision as to implement thrombolytic therapy or not. Other recent clinical practice guidelines [3] confirm that thrombolytic therapy is the first-line intervention in people with highrisk pulmonary embolism and cardiogenic shock and/or arterial hypotension. The picture is less clear with regard to intermediate-risk pulmonary embolism patients, in whom the routine use of thrombolysis is not recommended by the authors of the latter guidelines, even if it "may be considered in selected patients with intermediate-risk pulmonary embolism and after thorough consideration of conditions increasing the risk of bleeding".

Being a medical doctor is not an easy task, and nobody has ever said the contrary. It is typical of physicians, and in general of health operators, to work in conditions of uncertainty; yet physicians have to constantly make decisions in the interest of their patients. To do that, they formulate qualitative and quantitative estimations, also using, as in this therapeutic scenario, helpful instruments including clinical guidelines. Biomedical research is neither complete nor unambiguous, and consequently published literature does not provide the direct answer to every clinical question; black and white are not the dominant colours of the clinical spectrum, commonly characterized by multiple degrees of grey. Clinical practice guidelines, although always improvable, are already today supporting tools for decision making; they do not and they cannot replace human medical decisions. They are of value, as in this case, in recalling and underlining the opportunity and necessity of pondering in an integrated manner the clinical severity, the rationale prognosis and the accurate evaluation of the bleeding risk of the single patient so as to reach the best possible medical decision for the individual person. Without substituting themselves to patients (since they are not their patients) physicians are called upon to decide, not infrequently in a very short time, with their patients, whose values and preferences are, and should always be considered, essential pieces of the whole clinical puzzle by health professionals [8].

Conflict of interest statement The authors declare that they have no conflict of interest related to the publication of this manuscript.

\section{References}

1. Oxman AD, Chalmers I, Liberati A (2004) A field guide to experts. BMJ 329:1460-1463

2. Kearon C, Kahn SR, Agnelli G et al (2008) Antithrombotic therapy for venous thromboembolic disease: American College of Chest Physicians evidence-based clinical practice guidelines (8th edn). Chest 133:454S-545S

3. Torbicki A, Perrier A, Konstantinides S et al (2008) Guidelines on the diagnosis and management of acute pulmonary embolism: the Task Force for the Diagnosis and Management of Acute Pulmonary Embolism of the European Society of Cardiology (ESC). Eur Heart J 29:2276-2315

4. Dong B, Jirong Y, Liu G et al (2006) Thrombolytic therapy for pulmonary embolism. Cochrane Database Syst Rev CD004437

5. Jerjes-Sanchez C, Ramirez-Rivera A, de Lourdes Garcia M et al (1995) Streptokinase and heparin versus heparin alone in massive pulmonary embolism: a randomized controlled trial. J Thromb Thrombolysis 2:227-229

6. Meyer G PEITHO Pulmonary embolism thrombolysis studyprotocol. Available at: http://clinicaltrials.gov/ct2/show/NCT00 639743?term=pulmonary+embolism+tenecteplase\&rank=3

7. Konstantinides S (2008) Clinical practice. Acute pulmonary embolism. N Engl J Med 359:2804-2813

8. Conti AA, Gensini GF (2008) Doctor-patient communication: a historical overview. Minerva Med 99:411-415 